\title{
El suplemento de la imaginación en la narración. O de cómo Husserl aporta un complemento a la perspectiva de Ricoeur
}

Pol VANDeVElde

Marquette University

Philosophy Department

Milwaukee, WI 53201-1881 (Estados Unidos)

pol.vandevelde@marquette.edu

Abstract: I apply Edmund Husserl's notion of "phantasma", which he sees as the support for pure imagination, to Ricoeur's understanding of a narrative of real facts or events. I argue, first, that the phantasma, which plays in pure imagination the same role as sensations in perception, allows us to visualize and experience what is recounted in a narrative; and, second, that this phantasma is analogous to the sensations of perceptions that observers had of these facts and events. This component of imagination in a narrative is precisely what allows a narrative to render facts and events "as they really happened."

Keywords: Imagination, narrative, phantasma, Husserl, Ricoeur.
Resumen: Empleo la noción de "phantasma" de Edmund Husserl, que él ve como el soporte para la imaginación pura, a la interpretación de Ricoeur sobre la narrativa de hechos reales o acontecimientos. En primer lugar, sostengo que el phantasma, que desempeña en la imaginación pura el mismo papel que las sensaciones en la percepción, nos permite visualizar y experimentar lo que es relatado en una narración; y, en segundo lugar, que este phantasma es análogo a las sensaciones de las percepciones que los observadores tuvieron de estos hechos y acontecimientos. Este componente de la imaginación en la narración es precisamente lo que permite a ésta representar hechos y acontecimientos "tal como ellos realmente sucedieron".

Palabras clave: Imaginación, narrativa, phantasma, Husserl, Ricoeur. 
n este artículo, me propongo mostrar el rol que asume la imaginación en la narración de hechos reales, como son los testimonios o los relatos históricos, entre los cuales no haré aquí ninguna distinción. Paul Ricoeur ya se ocupó de este problema en su teoría de la narración y demostró que los hechos "tal como realmente sucedieron" no se pueden contar sino haciendo intervenir tres géneros: lo Mismo (lo que el relato narra es lo mismo que sucedió), lo Otro (la narración no es en sí misma el hecho que ésta tiene como su objeto), y lo Análogo (la narración es un equivalente del acontecimiento, del que es su "representación"). Por su parte, Edmund Husserl nos permitirá comprender mejor cómo funcionan estos tres géneros en la narración. Husserl formula la noción de phantasma para denominar al soporte de la imaginación; ello, por analogía con la percepción, que necesita del soporte de las sensaciones para hacer presente un objeto. Así, por ejemplo, cuando me imagino un centauro, un phantasma participa de la fantasía de igual modo que las sensaciones intervienen cuando percibo un mirlo emprendiendo el vuelo.

En lo siguiente, quisiera mostrar cómo en la narrativa también participa un phantasma que nos permite visualizar lo que cuenta el relato y, de este modo, nos permite hacer experiencia de lo narrado bajo el modo del "como si". Por este motivo, considero que no habría una diferencia radical entre percepción e imaginación, pues ambas incluyen un momento de lo que Husserl llama "mera presentación" por medio de sensaciones (en la percepción) o de phantasmas (en la fantasía). El aporte del phantasma a la narración consiste en que ésta última ya no cabrá ser leída simplemente como un recuento de información, sino que la narración cautiva la imaginación de los lectores de tal modo, que se ven envueltos en y por el relato, y hacen parte de la experiencia de lo acontecido "como si" ellos estuvieran allí. Por lo tanto, el género de lo análogo hace intervenir la imaginación en dos niveles: por un lado, el del autor de la narración, que debe hacer que los hechos y acontecimientos sean vívidos y - en tanto convincentes - reales; y, por el otro, el nivel de los lectores, quienes son invitados a hacer parte de la representación que ofrece el relato. 
En su libro La escritura o la vida, Jorge Semprún, sobreviviente del campo de concentración de Buchenwald durante la Segunda Guerra Mundial, cuenta que los prisioneros, todavía en los campos, se preguntaban acerca de cómo podrían hacerse comprender una vez fueran liberados, cómo poder contar los horrores a los que estaban sometidos, cómo, en suma, poder hablar de su "experiencia”. Semprún escribe: “¿Cómo contar una historia poco creíble, cómo suscitar la imaginación de lo inimaginable si no es elaborando, trabajando la realidad, poniéndola en perspectiva? ¡Pues con un poco de artificio!”'. Hace falta por tanto - como algunos prisioneros concluyen con Semprún-, apelar a otro tipo de comprensión por medio de la "escritura literaria", del "artificio de la obra de arte" 2 . El elemento ficcional e imaginativo serviría para restituir y traducir la brutalidad de lo real, la cual, sin este elemento ficcional, sería mera brutalidad sin relevancia y no lograría cautivar al interlocutor; en resumen: no cumpliría su propósito de expresar el horror. El temor de muchos supervivientes - si bien esto vale para cualquier víctima - no es solo que los hechos padecidos no sean contados; también es que las personas que leerán los reportes y los relatos no puedan percatarse de lo que significa sufrir lo que allí se les narra, que no alcancen a imaginar cómo era estar en un campo de concentración. Como le dice uno de los prisioneros, los testimonios, los relatos históricos contarán la verdad, pero echarán en falta la "verdad esencial", que no es "transmisible" . La imaginación sería así necesaria para las narraciones que cuentan hechos reales a fin de conferirles no solamente veracidad, sino también este poder de producir en los lectores una experiencia en el modo del "como si". Este suplemento de imaginación necesario para presentar lo real está en el centro de las investigaciones de Paul

1. J. SEMPRún, L'écriture ou la vie (Éditions du Seuil, Paris, 1994) 166; trad. cast.: La escritura o la vida (Fábula Tusquets, Barcelona, 1997) 141.

2. J. SEMPRÚN cit., 167; trad. cast., Ibidem.

3. J. SEMPRún cit., Ibidem; trad. cast., Ibidem. 
Ricoeur acerca de la narración, ya sea a propósito de la acción ${ }^{4}$, del tiempo ${ }^{5}$ o de la identidad del yo ${ }^{6}$.

\section{RICOEUR: EL SUPLEMENTO DE LA IMAGINACIÓN EN LA NARRACIÓN}

Ricoeur ha empleado diferentes enfoques para intentar explicar esta relación entre, por un lado, el orden de la acción y los hechos; y, por el otro, el orden de la narración. Para él, se trata de mostrar que la narración no es ni violenta con relación a la acción o los hechos (esto es, que no daría cuenta de ellos tal como fueron), ni redundante (apenas un simple apéndice de lo que ya fue totalmente articulado en el dominio físico de los gestos y movimientos). Ricoeur incluso llegó a afirmar en los tres volúmenes de su obra Tiempo y narración que el mundo de los hechos y las acciones ya está articulado a la manera de un relato. Ricoeur habla de una triple mímesis, cuyo primer nivel es el del mundo de los hechos y la acción. En este sentido, habla de la acción como una historia potencial o de una estructura pre-narrativa de la experiencia.

Sin embargo, una ambigüedad fundamental permanece, limitando toda la teoría ricoeuriana de la narración y que él mismo reconoce de manera implícita en sus formulaciones. Por ejemplo, en el capítulo "La imaginación en el discurso y en la acción", incluido en su obra Del texto a la acción, menciona tres roles que la ficción puede desempeñar en la narración de la acción: "[Y] a sea que la ficción contribuya a redescribir la acción que ya está presente, ya sea que se incorpore al proyecto de la acción de un agente individual, o bien que engendre el campo mismo de la acción intersubjetiva" ". En su primer

4. P. Ricoeur, Du Texte à l'action (Éditions du Seuil, Paris, 1986); trad. cast.: Del texto a la acción. Ensayos de hermenéutica II. $2^{\mathrm{a}}$ ed., trad. de Pablo Corona (Fondo de Cultura Económica, Ciudad de México, 2002).

5. P. Ricoeur, Temps et récit (Éditions du Seuil [Points], Paris, 1983 [Vol. 1], 1984 [vol. 2], 1985 [vol. 3]); trad. cast.: Tiempo y narración (Siglo XXI, Ciudad de México, 1995 [vol. I], 1995 [vol. II] y 1996 [vol. III]).

6. P. Ricoeur, Soi-même comme un autre (Éditions du Seuil, Paris, 1990); trad. cast.: Sí mismo como otro (Siglo XXI, Madrid, 1996).

7. P. Ricoeur, L'imagination dans le discours et dans l'action, en Du texte à l'action cit., 
rol, la ficción no opera sino en el nivel de la "redescripción" de la acción o acontecimiento y "redescribir — dice Ricoeur- es aún describir", puesto que el referente de la historia no es otro que "la acción ya dada allı". En sus funciones segunda y tercera, la ficción también opera a nivel del poder del sujeto, ya sea individual — esta es la segunda función — o colectivo - tercera función-, en el nivel del "yo puedo" de la acción y los proyectos. En estas tres funciones, la imaginación no interviene en el nivel de los hechos y los acontecimientos.

Sin embargo, en su ensayo "La función de la ficción en la conformación de la realidad", Ricoeur considera un elemento imaginario en la propia narración que va más allá de la re-descripción y del poder-hacer de los sujetos. Al decir que los sistemas simbólicos "hacen" y "rehacen" el mundo, Ricoeur parece conferir un peso ontológico a la narración, que consiste precisamente en "rehacer" y no solamente en "redescribir" la realidad. Quisiera reducir esta tensión entre "redescribir" y "rehacer" apelando a Husserl y su tratamiento de la imaginación, que el propio Ricoeur particularmente discute en La memoria, la bistoria, el olvido ${ }^{10}$.

Siguiendo a Husserl, Ricoeur distingue entre la imaginación reproductiva (que, como en el caso de la imagen, parte de un original que es luego reproducido) y la imaginación productiva (como es el caso de la ficción, que propone una nueva realidad sin anclaje en un original a ser reproducido). La imaginación productiva propone, por ejemplo, en términos de Ricoeur, "objetos no reales" o "una visión ampliada de la realidad"11. En una asertiva nota del curso sobre la imaginación que Ricoeur impartió en la Universidad de Chicago en 1975, éste afirma: "La imaginación no es en absoluto una alter-

213-214; trad. cast.: La imaginación en el discurso y en la acción, en Del texto a la acción cit., 197.

8. Ibidem, 223; trad. cast., 206 (énfasis de Ricoeur).

9. P. Ricoeur, The Function of Fiction in Shaping Reality, "Man and World" 12/2 (1979) 123-141.

10. P. Ricoeur, La mémoire, l'bistoire, l'oubli (Éditions du Seuil, Paris, 2000); trad. cast. La memoria, la historia, el olvido. Trad. de Agustín Neira (Trotta, Madrid, 2003).

11. "An expanded vision of reality" (P. RICOEUR, The Function of Fiction in Shaping Reality cit., 128). 
nativa a la percepción, sino un ingrediente de la percepción. Ella se aloja al interior del marco de la percepción"12.

Es posible observar la tensión entre estas diferentes funciones de la imaginación, así como el riesgo de conceder peso ontológico a la ficción. La tensión radica en las formulaciones de Ricoeur: de un lado, él considera al relato algo más que una narración posterior a un hecho, pero, por el otro, no puede poner demasiado en cuestión el estatuto de lo real, dado que lo real sirve de base para toda narración. El riesgo consiste precisamente en quitarle a lo real, para decirlo en términos de Sartre, su "coeficiente de adversidad"; es decir, la capacidad de lo real para resistir a las narraciones y para servir de árbitro entre relatos verídicos e historias fraguadas. Si, como Ricoeur, se quiere conferir a la historia más que un rol que el de la redescripción de la realidad o de la modelización de las capacidades del sujeto para hacer y actuar - he aquí las tres funciones antes mencionadas-, me parece inevitable asumir este riesgo y poner en cuestión la noción de realidad tal como es entendida en el sentido común. El punto de vista del sentido común considera la realidad como un conjunto de entidades claramente demarcadas, como los perros y los gatos, los campos magnéticos y las obras de arte. Poner en cuestión el sentido común conlleva reconocer que la realidad solo se da en las intenciones de conciencia (para la fenomenología) o las experiencias sensibles y los experimentos (para los enfoques empíricos, por ejemplo, los científicos). No se trata de poner a la ficción al mismo nivel que lo real o de eliminar sus diferencias, sino de reexaminar los criterios y procedimientos $-\mathrm{O}$, simplemente, los presupuestos $\mathrm{y}$ prejuicios-que permiten separar lo real de la ficción. Comencemos por las sutilezas del problema tomando a Ricoeur como guía, quien observó lúcidamente los límites de su modelo narrativo.

Ricoeur formula lo que él llama los tres géneros de lo Mismo, lo Otro y lo Análogo para denominar a lo que sucede entre la acción o el acontecimiento, por un lado, y la narración de esta ac-

12. "Imagination is not at all an alternative to perception but an ingredient of perception. It's encapsulated within the framework of perception", citado en G. H. TAYLOR, Ricoeur's Philosophy of Imagination, "Journal of French Philosophy" 16 (2006) 94. 
ción o de este acontecimiento, por el otro ${ }^{13}$. Ricoeur quiere mostrar cómo la narración puede verter los hechos y los acontecimientos tal como estos "verdaderamente sucedieron", según la frase de Leopold Ranke que a Ricoeur le gusta repetir. Los géneros de lo "Mismo" y de lo "Otro" realmente no ofrecen dificultades conceptuales para entender lo que Ricoeur quiere expresar. Por un lado, la narración dice "la misma cosa" que lo sucedido. Esto es lo que distingue a la historia o al periodismo de la literatura o la invención. Un historiador perdería su credibilidad al decir cosas falsas, al igual que un periodista perdería su trabajo fraguando historias. Por otro lado, la narración utiliza medios lingüísticos, estilísticos o retóricos que no se encuentran en el acontecimiento o la acción y permite urdir la trama textual a partir de la materialidad de los gestos y movimientos de la acción. La narración es, pues, un "otro" del acontecimiento o de la acción: es "diferente". Las complicaciones comienzan en el tercer género, lo Análogo. ¿Cómo una narración — hecha de palabras y frases, usando metáforas y otras figuras de estilo, y empleando medios retóricos para mantener al lector en suspenso y convencerlopuede dar cuenta de algo material como gestos y movimientos? ¿Cómo se puede pasar del nivel físico de la acción y el acontecimiento al nivel semántico del dar cuenta de este acontecimiento o acción sin pérdida ni violencia? ¿Cómo preservar la "mismidad" de lo real y de la narración manteniendo la diferencia de sus materias y de sus modos de operación?

Como es sabido, Ricoeur intentó en un inicio pensar la acción como un texto, haciendo de la legibilidad el método retrospectivo para discernir lo significado por la acción: ésta (la acción) ha de ser tratada como algo a ser leído (un texto). Sin embargo, este método retrospectivo parece querer imponer una categoría hermenéutica sobre la acción y el acontecimiento, como si, en el momento de la acción, los agentes operaran en función de la interpretación posterior de sus hechos y gestos - post factum-, esto es, como si ellos mismos estuvieran ejecutando una interpretación de eso que hacen en el momento en que lo hacen. La triple mímesis del primer vo-

13. Ver Temps et récit 3 cit., 272s; trad. cast.: Tiempo y narración III cit., 909ss. 
lumen de Tiempo y narración procuró otra aproximación bastante notable: el mundo de la vida y de la acción ya está estructurado por una forma narrativa (una Mimesis I, que es una "pre-figuración”), si bien la narración de la vida y de la acción (la Mimesis II como "configuración"), no hace sino hacer explícito lo que ya estaba implícito en el primer nivel, el de la vida y la acción. Ricoeur añadió una Mimesis III, que es una "re-figuración" en el sentido de un retorno del momento narrativo de la Mimesis II al mundo de la vida y de la acción (Mimesis I), pero, como él afirma, a un grado más elevado ${ }^{14}$.

Ricoeur detecta esta "pre-figuración” narrativa del mundo de la vida por el hecho de que la acción tiene una estructura temporal - tiene un inicio, un medio y un final- y de que existe una simbología de la acción: ciertos gestos están socialmente codificados y permiten a los otros anticipar lo que alguien irá a hacer. Por ejemplo, veo a alguien caminando lentamente por la noche cerca de mi edificio; se detiene, vacila, mira a derecha e izquierda. Intento predecir lo que está haciendo y lo que hará, considerando, en mi inquietud, diferentes escenarios: busco la legibilidad de su acción. Luego veo un pequeño perro con correa y recobro la calma al tratarse de un escenario familiar. Aparte de esta simbología de la acción, también hay una semántica de la acción que la estructura en términos de agente (que responde a la pregunta: “¿Quién?”), paciente (“¿Con quién o contra quién?"), motivo (“¿Por qué?”), modo (“¿Cómo?”), etc. ${ }^{15}$ Evidentemente, estas estructuras temporal, simbólica y semántica preceden al sujeto que actúa en el momento en que actúa y, de esta forma, le procuran un registro para realizar su acción. Esto fue lo que condujo a Ricoeur a hablar de la legibilidad de la acción o de historia (narración) potencial.

No obstante, hay dos preguntas que permanecen abiertas con relación al movimiento entre Mimesis I, Mimesis II y Mimesis III. Ricoeur bien afirma que este movimiento no es circular, sino en

14. Esta misma cuestión la he tratado anteriormente en P. VANDEVELDE, The Challenge of The 'Such As It Was': Ricoeur's Theory of Narratives, en D. KaPlan (ed.), Reading Ricoeur (State University of New York Press, Albany, 2008) 141-162.

15. Cf. P. Ricoeur, Life in Quest of Narrative, en D. Wood (ed.), On Paul Ricoeur: Narrative and Interpretation (Routledge, London, 1991) 28. 
espiral. No explica, sin embargo, ni qué implica el movimiento en espiral de la Mimesis ni cómo el tránsito de la Mimesis I (el mundo de la vida y de la acción) a la Mimesis II (el mundo de la narración) constituye la narración de lo que realmente aconteció, en vez de ser una invención o un revisionismo histórico.

Estas dos preguntas son en realidad dos problemas. El primero es el del ímpetu del movimiento que parte de la acción para desembocar en la narración, o que retorna desde la narración hacia la acción. El segundo problema es el de la fidelidad de la narración a la acción o acontecimiento. Estos dos problemas recargan el momento de lo Análogo, imponiéndole un enorme trabajo explicativo. Así pues, uno puede preguntarse en qué sentido la narración es "análoga" a lo real o cuál es el logos del analogon que es la narración. Ricoeur reconoció posteriormente que hace falta introducir un momento que yo denomino "existencial" en el proceso de Mimesis: la deuda y la atestación ${ }^{16}$. Es posible afirmar que este momento existencial da una respuesta al primer problema, el del ímpetu del movimiento narrativo, por ser lo que motiva (la deuda) y lo que justifica (la atestación) el gesto de contar un relato. Sin embargo, el segundo problema permanece intacto. ¿Por qué un tejido de palabras y frases, un relato o un texto, son capaces de traducir lo físico de la acción y el acontecimiento, de dar cuenta de ello, de "re-presentarlo" (como dice Ricoeur) restituyéndole lo adeudado?

Ricoeur inventa dos neologismos en francés para nombrar esta función notable que se le atribuye a la narración: "representancia" [représentance] y "lugartenencia" [lieutenance]. Ricoeur considera estos dos términos, especialmente el primero, como la traducción del término alemán Vertretung tal como fue usado por Karl Heussi. La narración hace las veces de la acción o del acontecimiento. Ella los representa en su ausencia, es, pues, su representante o su analogon. Sin embargo, como Ricoeur ya lo reconoce en el tercer volumen de Tiempo y narración, esta noción de representancia, una pala-

16. He examinado este problema en P. VANDEVELDE, Le fondement ontologique du récit selon Ricoeur: mimesis, dette et attestation, "Studia Phaenomenologica" XIII (2013) 244-259. 
bra francesa inexistente que debe traducir una palabra extranjera, es más el nombre del problema que su solución.

El problema es el siguiente: si solo se tiene la deuda y la atestación, entonces bien se podría prescindir del correlato: el hecho o la acción. Simplemente podría decirse que es a nivel intersubjetivo - en la conversación, como diría Richard Rorty, o en la comunicación, como dirían Jürgen Habermas y Karl-Otto Apel- que los hechos y las acciones recibirían su lugar y su sentido, y, por ello, su existencia en la esfera pública. Podría decirse que el hecho o la acción solo existen verdaderamente cuando son representados públicamente; que antes de esta articulación pública en una narración, y el reconocimiento de este relato como objeto de discusión pública, la acción o el acontecimiento, propiamente hablando, no existen y, por tanto, no tendrían ningún estatuto ontológico. Sin embargo, esta es una estrategia que Ricoeur rechaza ${ }^{17}$. El quiere preservar el acontecimiento como aquello que denomina, siguiendo nuevamente a Heussi, un Gegenüber: una "contrapartida", un vis-à-vis. Aun cuando el acontecimiento no puede ser aprehendido más que por medio de "hechos" que son construidos a partir de documentos, comprendidos y explicados por historiadores, y presentados como narraciones - las tres fases de la representación historiadora-, en la construcción histórica permanece un resto o excedente que asedia los hechos, permitiendo así tanto corregirlos como completarlos y reformularlos. Lo que ejerce tales restricciones sobre la narración es el acontecimiento como Gegenüber, que es como este horizonte "siempre en retirada" que Ricoeur menciona en La memoria, la historia, el olvido ${ }^{18}$. De este modo, Ricoeur preserva la posibilidad de la narración de "referir" algo que realmente sucedió, incluso si esta referencia es bastante indirecta, pasando por todo el trabajo de la evidencia y los documentos, de la comprensión/explicación y de la representación historiadora.

17. He abordado esta problemática en P. VANDEVELDE, The Enigma of the Past: Ricoeur's Theory of Narrative as a Response to Heidegger, en S. DAVIDSON, M.-A. Vallée (eds.), Hermeneutics and Phenomenology in Paul Ricoeur: Between Text and Phenomenon (Springer, Dordrecht, 2016) 123-139.

18. P. Ricoeur, La mémoire, l'bistoire, l'oubli cit., 593; trad. cast.: La memoria cit., 595. 
¿Cómo puede el analogon asumir este trabajo referencial, el cual, para Ricoeur, es deber referencial: "Toda la historia del sufrimiento clama venganza y pide narración"19. Esto es lo que él llama la "vehemencia asertiva de la representación historiadora" ${ }^{20}$. Ricoeur reconoce un momento de ficción en el analogon que permite pasar del nivel de los hechos y actos al nivel de las frases y narraciones - el tránsito de la Mimesis I a la Mimesis II-y, de modo inverso, de las narraciones a la acción —el tránsito de la Mimesis II a la Mimesis III-. Es posible decir que esta ficción en el núcleo del movimiento del analogon es la imaginación, por lo cual la imaginación es el logos del analogon: la imaginación "inventa" este analogon sin simplemente descubrirlo ni fraguarlo. La invención se sitúa en este ejercicio peligroso de gestionar, de un lado, el realismo de hechos y acontecimientos y, del otro, el idealismo de la narración o del texto, que bien podría prescindir de estos hechos "llamados" reales y presentar una realidad artificial.

Lo que propongo es hacer explícito lo que sugiere Ricoeur: que la imaginación no se encuentra solamente del lado de la narración con lo cual se pierde el contacto con la acción y el acontecimiento-, sino que la imaginación ya se encuentra también del lado de la práctica de la vida y de la acción. Como se mencionó previamente, Ricoeur tiende a subrayar el carácter retrospectivo de la narración. Por "retrospectivo" quiero decir que la narración hace explícito de manera retrospectiva — tras los hechos: post factum - aquello que se suponía implícito en la acción misma: por tanto, la acción como texto o como Mimesis I. Es por esto que Ricoeur habla de una "narratividad incoativa" en el mundo de la vida y de la acción ${ }^{21}$, de "relato potencial"22 o de "historias no narradas (todavía)"23, etc. La impresión que dejan estas formulaciones es que este lado narrativo implícito propio de lo real es, de hecho, lo que la narrativa establece con posterioridad. La narración operaría bajo el modo de una reconstrucción con el riesgo de una ilusión retrospectiva: una vez

19. P. RicoeUR, Temps et récit 1 cit., 143; trad. cast.: Tiempo y narración I cit., 145.

20. P. Ricoeur, La mémoire, l'bistoire, l'oubli cit., 367; trad. cast.: La memoria cit., 374.

21. P. RicoeUR, Temps et récit 1 cit., 141; trad. cast.: Tiempo y narración I cit., 144.

22. P. RiCOEUR, Life in Quest of Narrative cit., 30.

23. P. Ricoeur, Temps et récit 1 cit., 141; trad. esp.: Tiempo y narración I cit., 144. 
que se tiene la narración de la acción o del acontecimiento, parece casi evidente que estos tienen una estructura narrativa. La analogía depende entonces solamente de la imaginación de quien la narra. Por lo tanto, a fin de evitar la ilusión retrospectiva y el aspecto constructivista de esta reconstrucción, es preciso demostrar que la imaginación no es post factum, que no solo está en vigor en la narración, sino que ella es, por así decir, intrínseca al hecho. Para que haya una verdadera analogía, hace falta que la acción o la vida misma involucren a la imaginación. Es preciso, por tanto, demostrar que la imaginación habita lo real. Es en este punto que Edmund Husserl puede ayudarnos. Desde luego, Ricoeur está familiarizado con la obra de Husser ${ }^{24}$. A continuación, seguiremos algunas observaciones de Husserl acerca de la imaginación y la fantasía ${ }^{25}$ que nos ayudarán a comprender cómo éstas ya se encuentran operando en nuestro acceso a lo real, por ejemplo, en el caso de la percepción.

\section{HusserL: LA IMAGINACIÓN EN EL NÚCLEO DE LA PERCEPCIÓN}

En su filosofía, Husserl apela en varias ocasiones a la imaginación. La imaginación interviene, por ejemplo, en el proceso de reducción

24. Ricoeur tradujo al francés Ideas I y ha estado, como afirma en el título de uno de sus libros, En la escuela de la fenomenología [A l'école de la phénoménologie]. Él emplea también las reflexiones de Husserl en su obra La memoria, la historia, el olvido.

25. N.d.T.: Si bien en francés el vocablo "imagination" ha venido siendo empleado para traducir el término alemán Phantasie, considero importante distinguir ambos términos. La distinción entre imaginación y fantasía reside en el carácter rigurosamente técnico que para Husserl posee esta última. Dicho bastante esquemáticamente, la fantasía (Phantasie) es un género de actos intuitivos objetivantes de igual legitimidad que la percepción y que, a diferencia de esta última, se caracteriza porque sus contenidos son dados "como si fueran presentes" [als ob], esto es, son dados bajo la forma de una "presentificación" [Vergegenwärtigung]. Los actos intuitivos de la fantasía pueden ser posicionales o no posicionales, y tiene como sus subespecies o modos a la rememoración (los recuerdos en sentido ordinario), la expectativa, la empatía, la conciencia de imagen y la "fantasía pura". Todos ellos son formas de la fantasía. Como se puede apreciar, la fantasía concierne más a una modificación de los contenidos hiléticos (de allí la importancia de la oposición entre presentación y presentificación) sobre los que se funda un objeto, que si algo es traído a la conciencia como una imagen (visual). En este sentido, la noción de "imaginación" que emplea el autor de este artículo se aproxima al sentido filosófico que, en alemán, posee el término Einbildungskraft. 
dirigido, a la vez, hacia objetos y hacia los otros. La "libre variación" de la fantasía, en efecto, permite al fenomenólogo hacer variar los datos de una cosa a fin de encontrar su "esencia"; esto es, que es posible ver su esencia ideal e invariante más allá de la existencia de la cosa. Como Husserl afirma, la ficción hace parte del método fenomenológico. Él escribe: "Así se puede decir realmente, si se ama el lenguaje paradójico, y decir con estricta verdad, con tal de que se entienda bien el equívoco sentido, que la 'ficción' constituye el elemento vital de la fenomenología, como de toda ciencia eidética; que la ficción es la fuente de donde saca su sustento el conocimiento de las 'verdades eternas"'26. La fantasía también opera en la empatía al permitir tener acceso a las experiencias de los otros: imaginariamente me transpongo en su situación; luego, yo podría hacer experiencia de lo que el otro siente. Esta transposición por analogía me confiere un "como si" que me permite ampliar las fronteras de mi experiencia original. La fantasía, en ambos casos, en la variación eidética y en la experiencia de otros, produce "irrealidades" y son precisamente estas irrealidades las que dan acceso a algo como la realidad. Sin embargo, al igual que en Ricoeur, este papel de la imaginación no se limita únicamente al discurso descriptivo del fenomenólogo. En el volumen XXIII de la colección Husserliana: Fantasía, conciencia de imagen, recuerdo [Phantasie, Bildbewusstsein, Erinnerung $]^{27}$, Husserl va incluso más allá y pone en cuestión la estricta separación de la percepción y la imaginación. En lo que sigue, solo emplearé algunas de sus observaciones para mostrar que ya hay un momento imaginativo en el núcleo mismo de actos de conciencia tales como la percepción.

En términos generales, Husserl entiende la fantasía como una "presentificación" o un "hacer presente" (Vergegenwärtigung) por

26. E. HusserL, Idées directrices pour une phénoménologie, vol. I, \$70, trad. P. Ricoeur (Gallimard, Paris, 1950) 227; trad. cast.: Ideas relativas a una fenomenología pura y una filosofía fenomenológica. Libro Primero: Introducción general a la fenomenología pura. Trad. de A. Zirión (Fondo de Cultura Económica, Ciudad de México, 2013) 235. (Énfasis de Husserl).

27. E. Husserl, Phantasie, Bildbewusstsein, Erinnerung. Zur Phänomenologie der anschaulichen Vergegenwärtigung. Texte aus dem Nachlass (1898-1925). Husserliana XXIII. Eduard Marbach (ed.) (Martinus Nijhoff, Den Haag, 1980). En adelante, Hua XXIII. 
oposición a una "presentación” (Gegenwärtigung), que es, por ejemplo, el caso de la percepción. La "presentificación" generalmente es caracterizada como una modificación de la "presentación" y, por tanto, se encuentra basada en ella. Dado que la fantasía "trae al presente" o "presentifica" lo que la percepción "presenta", ella es "reproductiva". Husserl caracteriza la "presentificación" (Vergegenwärtigung) como una "intencionalidad modificante" (modifizierende Intentionalität $)^{28}$, donde el acto de fantasía constituye su objeto por modificación, produciendo "cuasi' una objetualidad" ("quasi” eine Gegenständlichkeit), en el sentido de una objetualidad posible (mögliche Gegenständlichkeit). Podría decirse que esta "cuasi objetualidad" posee dos valores ontológicos. El primer valor la liga a la presentación original. La fantasía que produce posibilidades está, de cabo a rabo, en relación con la realidad efectiva e interactúa con ella. "Toda objetualidad fantaseada (posibilidad objetiva y pura) remite a una conciencia constituyente bajo el modo del cuasi, una conciencia con un yo consciente, $\mathrm{y}$, al mismo tiempo, remite a un sujeto fantaseante, que es un sujeto realmente efectivo con una vivencia realmente efectiva al cual pertenece el fantasear mismo"29. El "cuasi" designa entonces una superestructura anclada en la presentación original y dependiente de ella.

El segundo valor ontológico es más interesante. Uno puede fantasear algo que no tenga anclaje en la percepción. En este caso, debemos reconocer, como lo hace Husserl, que la presentificación puede ser una intencionalidad por sí misma y, por tanto, independiente de la percepción. En la pintura abstracta, por ejemplo, o en una fantasía pura (como sería imaginar tener otro tipo de vida profesional), ya no hay dependencia de la fantasía respecto de la percepción; de hecho, se tiene incluso una relación inversa. En vez de partir de la percepción para modificarla en la fantasía, es la fantasía la que propone una nueva percepción o la percepción de aquello que

\section{Hua XXIII, 566.}

29. "Jede phantasierte Gegenständlichkeit (gegenständliche reine Möglichkeit) weist zurück auf ein es quasi konstituierendes Bewusstsein mit Bewusstseins-Ich, und zugleich auf ein phantasierendes Subjekt, das wirkliches Subjekt ist mit wirklichem Erleben, wohin das Phantasieren selbst gehört" (Hua XXIII, 567). 
no es estrictamente perceptible. Estamos hablando entonces de una fantasía productiva y ya no de una reproductiva.

Si la fantasía puede producir una percepción, en la percepción misma debe haber una ausencia que se puede completar en la conciencia $y$, entonces, ha de haber componentes que no son dados en presente (presentados), pero que, no obstante, contribuyen a la presentación. Husserl reconoce tal ausencia. En primer lugar, está el hecho bien conocido de que al ver un objeto físico (por ejemplo, un árbol) uno no ve únicamente la parte frontal del árbol, sino el árbol mismo. En otras palabras, la parte posterior del árbol también me ha de ser dada de cierta manera: es "apercibida", como dice Husserl, en tanto es percibida al mismo tiempo. Desde luego, en este caso, se podría decir con Husserl que esta apresentación es diferente a la imaginación, pues no hay un segundo acto que tiene lugar después del primer acto de percibir la cara frontal del árbol. Más bien, es posible decir que se trata de un momento estructural de la percepción que forma parte de sus mecanismos, ya como automatismo, ya como proceso fisiológico.

Esta explicación sería plausible si esta apercepción no funcionara más que al nivel elemental de la cosa física, tal como sería percibir en tres dimensiones o distinguir una superficie de un volumen. Sin embargo, la apercepción también funciona en el caso de los aspectos culturales. Yo percibo directamente, dice Husserl, una antigua piedra miliar romana ${ }^{30}$. Partiendo de la cosa física, no hay inferencia al añadir, poco a poco, rasgos culturales e históricos: es una piedra, es antigua, está grabada, está en latín, es romana, etc. De una vez, en mi simple percepción, veo una antigua piedra miliar romana en toda su complejidad histórica y cultural. Husserl afirma que estos rasgos percibidos a la vez en la percepción, pero no dados directamente en términos de datos sensibles, nos son dados a través de lo que él llama "intenciones significativas" ${ }^{1}$. Esto no quiere

30. Cf. E. Husserl, Logische Untersuchungen. Zweiter Band, zweiter Teil: Untersuchungen zur Phänomenologie und Theorie der Erkenntnis, ed. U. Panzer (Martinus Nijhoff, Den Haag, 1984) [Hua XIX/2], 592; trad. cast.: Investigaciones lógicas, vol. 2. Trad. de M. G. Morente, J. Gaos (Alianza Editorial, Madrid, 1999) 641.

31. He analizado esta problemática en P. VANDEvelde, How Husserl's and Searle's 
decir, por lo demás, que el acto de percepción vaya acompañado de otros actos que serían "presentificaciones" del miliario romano. Estas "intenciones significativas" habitan en mi percepción; además, redefinen la relación entre la presentación original (Gegenwärtigung) y presentificación (Vergegenwärtigung), que no es una relación estática y cronológica, sino que también indica la presencia en el núcleo de la percepción de lo que bien debe denominarse fantasía. Si percibo inmediatamente una antigua piedra miliar romana, todas mis lecturas sobre la antigua Roma, los cursos que seguí, etc. "informan" mi percepción, dándole su carácter sofisticado. Veo el miliario con todos sus momentos imaginativos o imaginantes [imaginants] provenientes de mi cultura.

Husserl presenta otros dos casos interesantes de percepción en los que interviene un momento imaginativo. Uno de ellos es lo que él denomina "conciencia de imagen" (Bildbewusstsein); otro, el caso de la imaginación pura (Phantasie). La conciencia de imagen presupone la percepción de un soporte físico (das Bild als physisches Bild) - por ejemplo, un lienzo pintado- que es dado a la conciencia en el modo perceptivo; seguidamente, hay una "imagen mental" (geistiges Bild) u objeto-imagen (Bildobjekt), que consiste en las formas y colores del lienzo. Normalmente, esta imagen no es percibida por sí misma, sino como una manifestación (Darstellung) de otra cosa. Por último, está también la percepción de aquello que es figurado por medio de la imagen, a lo que Husserl denomina tema-de-laimagen (Bildsujet) ${ }^{32}$. Desde luego, uno puede poner atención en el soporte físico (como la textura de la tela) y puede prestar atención a los trazos violentos sobre el lienzo de una pintura de Van Gogh, pero la mayoría de las veces uno vive en la experiencia de la tela y yo "veo" (percibo) aquello que se presenta: por ejemplo, un campo de girasoles.

Contextual Model Reformulates the Discussion about the Conceptual Content of Perception, en R. Walton, S. Taguchi, R. Rubio (eds.), Experiential Reason. Husserl on Perception, Affectivity, and Volition. (Springer, Dordrecht, 2016) 57-76.

32. Hua XXIII, 21 ss. 
En un anexo a sus lecciones de 1904/190533, Husserl toma el ejemplo de una pintura de Tiziano. Él se pregunta: “¿Es el 'tema' un objeto que está representado como figura por medio de la imagen y debe valer como fundamento de una representación inauténtica?" 34. El peligro sería reduplicar lo que se representa: “¿Una otra intuición da una representación más auténtica de lo mentado en la conciencia de imagen estética?" 35 . Husserl afirma que es preciso concluir que lo que me interesa no es el objeto [de la representación] (exterior al lienzo), sino la manifestación de dicho objeto (Darstellung ${ }^{36}$ ) que es propia del lienzo. El interés recae "en el objeto-imagen siempre y cuando, en cuanto, y toda vez que éste traiga a intuición el tema-de-la-imagen" ${ }^{37}$. Husserl considera que "[l]a imagen [la imagen producida por Tiziano] no tiene la función de dirigirse a alguna 'otra cosa""38. Por el contrario, "[e]n el objeto-imagen veo el tema; vívidamente en sus trazos analogizantes, tengo una intuición, una conciencia analógica del objeto"39.

En este caso, tenemos tres actos intrincados unos con otros: (i) la conciencia perceptual del lienzo como un objeto físico, (ii) la conciencia de este lienzo como una manifestación de otra cosa y (iii) el reconocimiento de esa otra cosa que es exhibida y que constituye el tema de la pintura. Sin embargo, todos estos actos tienen lugar en términos de percepción y, mientras sigo la invitación que me hace la

33. N.d.T.: El autor hace referencia a las lecciones que Husserl impartió como tercera parte del curso del semestre de invierno del año 1904/1905, titulado Partes principales de la fenomenología y la teoría del conocimiento [Hauptstücke aus der Phänomenologie und Theorie der Erkenntnis]. Esta tercera parte estuvo dedicada a la fantasía y la conciencia de imagen. $C f$. Hua XXIII, Texto № 1, 1-108.

34. " $[\mathrm{I}]$ st das 'Sujet' ein Gegenstand, der durch das Bild als Abbild repräsentiert ist und <das als> Fundament für eine auf ihn bezügliche uneigentliche Vorstellung dienen soll?" (Hua XXIII, 154).

35. "Gibt eine andere Anschauung eine eigentlichere Vorstellung des im ästhetischen Bildbewusstsein Gemeinten?” (Hua XXIII, 154).

36. Hua XXIII, 155.

37. "[a]uf das Bildobjekt, sofern und soweit und so wie es das Sujet zur Anschauung bringt" (Hua XXIII, 154).

38. "Das Bild hat nicht die Funktion, etwas , anderes' vorstellig zu machen" (Hua XXIII, 155).

39. "Im Bildobjekt schaue ich das Sujet, in seinen analogisierenden Zügen lebend, habe ich eine Anschauung, ein analogisches Bewusstsein vom Objekt" (Hua XXIII, 154-155). 
pintura, mientras percibo la pintura, vivo en lo que es representado en ella o, en otras palabras, estoy ocupado en la conciencia de la ilusión. El lienzo hace "como si" exhibiera lo real.

El "como si" es una modalización de una percepción real, pero esta percepción no necesariamente debe preceder a la imaginación. Ella puede seguir a y ser el resultado de la imaginación. Incluso si el campo de girasoles representado es un campo análogo al que es posible percibir mediante los sentidos, e incluso si esta conciencia de imagen es, como tal, una presentificación que presupone o reproduce un acto de primer nivel (esto es, una percepción), esto solo es verdadero si adoptamos una perspectiva de reconstrucción explicativa para mostrar de qué manera el cuadro funciona como un cuadro pintado. Sin embargo, en el cuadro, yo sigo percibiendo directamente el campo de los girasoles sin que éste sea necesariamente tenido como la percepción de campos de girasoles reales. Se trata también, por tanto, de una percepción en la imaginación, la cual es hecha posible por la imaginación. La pintura abstracta lo demuestra. Ella no reproduce una primera percepción, incluso virtual, y no ofrece una ilusión en términos de cuasi-percepción de alguna cosa. Sin embargo, sigue siendo una percepción que desafía nuestro uso normal de la percepción en la medida en que las cosas y objetos son sustraídos, ya no se encuentran "representados".

Incluso si percibo formas y colores, e incluso si percibo la imagen de un campo de girasoles, hay otro componente que me permite vivir en una cuasi-percepción de un campo de girasoles que no es real en tanto que presente "en persona" [en chair et en os] —si cabe decir-, pero que no es simplemente una ausencia. Como dice Husserl, "[e]n la representación de fantasía, ciertamente tenemos la aparición de un objeto, pero no es la aparición de algo presente mediante el cual acaecería la aparición de algo "no presente" "40. Esto quiere decir que la pintura no es una imagen que representa un objeto, sino que funciona más bien como en el teatro, donde

40. "In der Phantasievorstellung haben wir zwar eine Erscheinung von einem Gegenstand, aber keine Erscheinung von einem Gegenwärtigen, mittels welcher Erscheinung von Nichtgegenwärtigem zustande käme” (Hua XXIII, 83). 
"[1]a representación (Darstellung) del actor no es una manifestación en el sentido en el que, hablando de un objeto-imagen, decimos que el tema de la imagen se manifiesta en éste" ${ }^{41}$. Es para explicar esta percepción en la fantasía que Husserl introduce la noción de phantasma, que dejaré sin traducir, a fin de mostrar una analogía entre la manera en que funcionan la percepción y la imaginación.

El phantasma se define como el "contenido sensible de la fantasía" ${ }^{2}$. El phantasma desempeña un rol análogo al que desempeñan las sensaciones en la percepción. La diferencia es que el phantasma no es concreto como las sensaciones: es "irreal". Cuando imagino a un centauro, tengo una imagen en mente, pero el soporte de esta imagen es no-sensible y, por tanto, "irreal". Si percibiera un centauro real, el soporte serían las sensaciones: "Frente a ellas [las sensaciones], los phantasmas son como nulidades. Ellos son irreales, no tienen ninguna validez en sí mismos, sino sólo como representante de otra cosa que, si fuera dado, justamente sería una sensación"43. Este calificativo de "irrealidad" (Irrealität) $)^{44}$ es interesante en dos aspectos. Por un lado, sugiere que hay algo en la imaginación que escapa a la percepción y que no es un producto directo. Uno puede imaginar alguna cosa sin modificar necesariamente una percepción de la misma (como en el caso del centauro).

Por otro lado, el segundo punto de interés proviene de una observación de Husserl, cuando sostiene que esta "irrealidad" recibe un "presente adquirido" (akquirierte Gegenwart) 4 . La presentificación puede producir un presente o una presencia que no es

41. "Die Darstellung des Schauspielers ist auch nicht eine Darstellung in dem Sinn, in dem wir von einem Bildobjekt sagen, dass sich in ihm ein Bildsujet darstelle" (Hua XXIII, 515). Marc Richir ha aplicado la noción de imaginación (fantasía) pura en el teatro y la novela en su artículo The Role of Phantasia in the Theatre and Novel, trad. J. Bechtel, en P. VANDEVELde (ed.), Phenomenology and Literature: Historical Perspectives and Systematic Accounts (Königshausen \& Neumann, Würzburg, 2010) 199-207.

42. "der sinnliche Inhalt der Phantasie" (Hua XXIII, 80).

43. "Ihnen gegenüber sind Phantasmen wie Nichtigkeiten. Sie sind irreal, sie gelten nichts für sich, sondern nur als Darsteller für anderes, das gegeben eben wieder Empfindung wäre" (Hua XXIII, 77).

44. Hua XXIII, 81.

45. Ibidem. 
simplemente la reproducción de la presencia real, sino un nuevo presente o una nueva presencia. Por lo tanto, la presentificación (Vergegenwärtigung) no es necesariamente dependiente de una presentación original (Gegenwärtigung) y no ha de ser necesariamente una reproducción. Si un cuadro me abre a nuevas percepciones, mi percepción del cuadro está en sí misma habitada por la imaginación: veo algo más que un lienzo, formas y colores; veo un campo de girasoles que no es real. De manera similar, leo Fausto y me veo fascinado por la historia; miro el filme Lincoln y vivo la vida de los personajes históricos representados. Percibo a Fausto o Lincoln más allá de la percepción de las letras en la página o en el grano del celuloide de la proyección, los cuales no veo la mayor parte del tiempo. Percibo una irrealidad porque veo lo que está representado y vive en esta representación "como si" fuera real. En la exhibición de un filme o en la lectura, "nosotros, que no somos niños" $"$, como afirma Husserl, "percibimos de modo activo, juzgamos de modo activo, cumplimos expectativas, esperamos y tememos, entristecemos y nos conmovemos de alegría, amamos y odiamos, etc. Pero todo esto 'en' la fantasía, en el modo del como-si”" Hay, por tanto, una "presencia adquirida" en la imaginación, tal y como hay una presencia imaginada en la percepción. En la imaginación, soy cautivado por la historia que leo o veo en el cine, participando en estos acontecimientos en el modo del "como si". En la percepción, "veo a la vez" los aspectos de las cosas que no se presentan directamente, como los rasgos culturales e históricos de una pintura de Van Gogh o de una piedra miliar romana.

46. "[W]ir, die wir keine Kinder sind" (Hua XXIII, 516).

47. "[W]ir nehmen wahr in aktiver Weise, wir urteilen in aktiver Weise, wir vollziehen Erwartungen, wir hoffen und furchten, wir trauern und sind freudig bewegt, wir lieben und hassen usw. Aber all das, 'in' der Phantasie, im Modus des Als-ob” (Hua XXIII, 517). Cf. igualmente el siguiente fragmento: "En la fantasía, lo intuido no es vuelto consciente por nosotros simplemente como realidad efectiva, como presente, pasado, etc. Lo intuido es vuelto consciente por nosotros con su contenido 'como si' fuera presente, etc. Es para nosotros una realidad efectiva "como si"" // ["In der Phantasie ist ein Anschauliches nicht als Wirklichkeit, als Gegenwart, Vergangenheit usw. schlechthin bewusst, sondern es ist uns bewusst mit seinem Inhalt, 'als ob' es gegenwärtig usw. wäre, es ist uns Wirklichkeit 'als ob’] (Hua XXIII, 504). 
Esta interacción entre la fantasía y la percepción desafía la estricta distinción entre la presentación (Gegenwärtigung) y la presentificación (Vergegenwärtigung). Husserl reconoce esta ambivalencia en un texto varias veces enmendado, donde considera la posibilidad de no oponer la percepción a la imaginación: "Percepción — fantasía no es la contraposición de presentación y presentificación [...]. La fantasía es como si fuera una presentación" ${ }^{48}$. Sin embargo, en el parágrafo inmediatamente siguiente, Husserl da marcha atrás y afirma: "El como si fuera es el carácter de la reproducción. El como si fuera percepción [es] el carácter de la fantasía en sentido estricto. Por ello se puede decir que la fantasía es ordinariamente un concepto más amplio = reproducción intuitiva" 49 .

El pasaje enmendado me parece más interesante y justo: al leer una obra o al ver un filme, percibo a Fausto o a Lincoln. Quisiera defender lo que Husserl sostiene en el fragmento posteriormente enmendado y sustentar esta defensa en lo que Husserl afirma en otro lugar: que la percepción y la fantasía tienen un soporte en común: las sensaciones y los phantasmas, respectivamente. Hay, pues, una “presentación” que es común a los dos actos y que precede a la toma de posición concerniente a la realidad del objeto (en la percepción) o su no-realidad (en la imaginación). Husserl llama a esta "presentación” una "blosse Vorstellung", una mera presentación ${ }^{50}$. Debe decirse

48. "Wahrnehmung - Phantasie ist nicht der Gegensatz von Gegenwärtigung und Vergegenwärtigung (...). Phantasie ist gleichsam Gegenwärtigung" (Hua XXIII, 269).

49. "Das Gleichsam ist der Charakter der Reproduktion. Gleichsam Wabrnebmung der Charakter der Phantasie im engeren Sinn. Doch kann man sagen, dass 'Phantasie' gewöhnlich ein weiterer Begriff ist = intuitive Reproduktion” (Hua XXIII, 269).

50. N.d.T.: A lo largo de este artículo, traduciré Vorstellung por "presentación" en vez del más usual "representación". Ello básicamente por dos razones. La primera es que, en alemán, el verbo vorstellen tiene la acepción de "presentar" (más evidente bajo la forma pronominal sich vorstellen, que se traduce como "presentarse", como cuando uno se presenta ante otra persona). La segunda razón es que, si bien el término "representar" es correcto y muy extendido en el vocabulario filosófico, en este contexto el prefijo "re-" induce a error y no alcanza a captar la sutileza técnica en juego. Una Vorstellung es aquí sencillamente una "presentación", como algo que se da inmediatamente en y para la conciencia. No se trata pues de ninguna representación, entendida ni como figuración, ni como segunda presentación (representar) ni como ocupar la función vicaria de algo ausente. 
entonces que la percepción y la fantasía son en sí mismas "modificaciones" de esta "mera presentación". Cuando las sensaciones se fusionan en un objeto y dan lugar a una "aprehensión" (Auffassung), acaece una mera "presentación". Es una Vorstellung, una mera presentación en tanto que un objeto es presentado en la conciencia pero sin que yo sea explícitamente consciente de ello. Esto todavía no es una Gegenwärtigung, una presentación directa a la conciencia que da lugar a una "conciencia de" (como en el ejemplo del mirlo que alza el vuelo, del cual estoy directamente puesto al tanto). La "mera presentación" (blosse Vorstellung) no contiene ninguna toma de posición (Stellungnabme) respecto de la existencia del objeto (en cuyo caso sería una percepción), de su inexistencia (entonces sería una fantasía) o de su pasado (ello sería un recuerdo). Lo que produce esta "mera presentación" son las sensaciones en la percepción o los phantasmas en la fantasía. En el volumen XXIII de la Husserliana, por ejemplo, Husserl sostiene que la "mera presentación" puede ser pensada "como un mero tener algo flotando frente a uno, como mirarlo sin tomar una decisión acerca de ello" ${ }^{51}$.

Si hay una "mera presentación" tanto en la percepción como en la imaginación, es necesario establecer una diferencia en la "creencia" que normalmente acompaña a la presentación como Gegenwärtigung. Husserl mismo reconoce dos tipos de "creencia". Un tipo es explícito. Se trata de la percepción de un objeto como tal, como una cuchara o una pintura, con su contraparte en la presentificación (en la imaginación) como no-creencia. El otro tipo de creencia es una creencia previa, que Husserl califica de "latente", como una mera presentación de algo en general tan pronto como hay una "aprehensión” de sensaciones (Auffassung) en la percepción o un phantasma (en la fantasía).

La creencia implícita o "latente" es lo que Hume denomina "belief" y constituye la idea formada sobre la base de una experiencia de nuestras sensaciones. Husserl se refiere explícitamente a esto:

51. "als blosses Vorschwebenhaben, darauf Hinblicken ohne jede Entscheidung" (Hua XXIII, 10). 
La posicionalidad es un modo esencial de vivencias intencionales que también constituye el belief latente. Ya que el belief (originariamente en Hume y Mill) debía expresar algo que pertenece al fenómeno independientemente del dirigirse-a o del no-dirigirse-a. La posicionalidad no es una creencia en sentido propio. Puesto que éste es el carácter fundamental de una cierta clase fundamental de tomas de posición ${ }^{52}$.

Antes de la percepción de una cosa como tal con su posicionalidad explícita (veo un mirlo emprendiendo el vuelo), hay una posicionalidad que aún no es explícita, que no es una toma de posición (hay un mirlo allí, frente a mî), sino una mera presentación (Vorstellen). Esta "mera presentación" puede ser un phantasma, con su creencia negativa ("eso no es real"), o una aprehensión de sensaciones (Auffassung) con su creencia positiva: "está efectivamente allí frente a mí”. A este respecto, Husserl afirma:

La creencia es aquí el modo originario de la serie de fenómenos de la "aprehensión" que denominamos aparición perceptiva $a^{53}$ de una cosa, etc. La creencia no es nada añadido a la percepción, sino percepción en el modo originario ${ }^{54}$.

52. "Die Positionalität ist ein Wesensmodus intentionaler Erlebnisse, die auch den latenten belief ausmacht. Denn der belief sollte etwas von Zuwendung oder Nichtzuwendung unabhängig zum Phänomen Gehöriges (ursprünglich bei Hume und Mill) ausdrücken. Die Positionalität ist nicht ein Glaube im eigentlichen Sinn. Denn das ist der Grundcharakter einer gewissen Grundklasse von Stellungnahmen" (Hua XXIII, 458).

53. N.d.T.: Es fundamental mencionar aquí que Husserl distingue entre dos modos de percepción: Wabrnebmung y Perzeption. En castellano, ambos términos se traducen igual ("percepción"). La diferencia radica en lo siguiente: la Wabrnebmung es la forma primaria de la experiencia perceptual ordinaria, la cual posee una inherente creencia empírica en la realidad sensible. Por su parte, la Perzeption (y su forma adjetiva, perzeptiv) se refiere exclusivamente a una modalidad de conciencia perceptiva vaciada del carácter ponente, es decir, neutralizada. En otras palabras: la Perzeption es el resultado de sustraer la toma de posición (Stellungnabme) a una percepción sensible (Wabrnebmung). A fin de distinguir ambos términos, emplearé exclusivamente cursivas ("percepción/perceptivo") para verter Perzeption/perzeptiv.

54. "Glaube ist also hier der Urmodus in der Reihe der Phänomene der ,Auffassung", die wir perzeptive Erscheinung eines Dinges u. dgl. nennen. Glaube ist nichts neben der Perzeption, sondern Perzeption im Urmodus" (Hua XXIII, 405). 
Debido a que hay este momento de "mera presentación" (blosse Vorstellung) en la base de la percepción y de la imaginación, la imaginación puede ser una "presentación" (Gegenwärtigung) sin pasar por la percepción a la que modificaría, como en el caso del centauro, pero también en la pintura y el teatro. De igual manera, cabría decir que la percepción puede ser una "presentificación" (Vergegenwärtigung) cuando está impregnada de cultura e historia. Cuando se reconoce que en la percepción hay una "mera presentación" como coalescencia de sensaciones en una "aprehensión" (Auffassung) y que esto aún no es una toma de posición, se comprende la legitimidad del término que Husserl emplea como sinónimo de "aprehensión": una "interpretación" (Deutung). No se trata de un acto explícito de interpretación que tomaría las sensaciones como objeto, sino, más bien, del hecho de que las sensaciones se hallan "unidas" de una cierta manera, en un cierto "sentido" (Sinn). Las manchas de colores, los sonidos, los movimientos se organizan en algo: "un mirlo que emprende vuelo". Aun cuando no se trata de un "acto" de interpretación, este momento de aprehensión o interpretación es una forma de articulación que abre la puerta a informaciones provenientes de otros horizontes. Aquello que hemos leído y aprendido nos hace más sensibles a ciertos aspectos de las cosas.

Cuando las sensaciones se fusionan en una "mera presentación”, esta coalescencia puede incluir un elemento de phantasma: por ejemplo, una percepción enriquecida por el arte de la pintura puede dar una perspectiva diferente; y esto, sin que el arte añada nada a las sensaciones externas, sino solo dando forma u organizando su aprehensión (Auffassung). A la inversa, la imaginación también puede neutralizar la "captación" de sensaciones, deconstruir la "mera presentación" del objeto y descomponerlo en sensaciones, que devienen entonces "presentes"; tal como expresó Cézanne cuando le dijo extasiado a su cochero: “¡Mira! ¡Los azules! Los azules allí bajo los pinos" $"$.

55. Citado en H. MALDINEY, Regard, parole, espace (L'Âge d'Homme, Lausanne, [1973] 1994) 138, citado igualmente por E. BEHNKE en On the Deep Structure of WorldExperiencing Life, "Études phénoménologiques-Phenomenological Studies" 2 (2018) 32 . 
Si se aplica esto a la narrativa, se observa mejor cómo la misma narración histórica (de hechos o acontecimientos) requiere de la imaginación. Para que las letras y las palabras produzcan imágenes y escenarios, se necesita un phantasma como medio que permita al relato representar un acontecimiento. El phantasma propio de la narración es análogo a la "aprehensión" (Auffassung) de sensaciones que tuvo lugar en la percepción "en directo" de este mismo acontecimiento por los primeros testigos. Esta percepción hace intervenir un momento imaginativo bajo la forma de una "organización" de sensaciones conformadas por el contexto histórico. Hay así un elemento imaginativo común que explica cómo la narración puede ser el analogon de un hecho o de un acontecimiento.

\section{CONCLUSIÓN}

Hablar, como Ricoeur, de "narratividad incoativa", de "relato potencial", de vida "en busca de narración" parece estar bien justificado. La anterior digresión en torno a la noción husserliana de phantasma explica también por qué el neologismo de "representancia" que emplea Ricoeur es bastante conveniente por dos motivos: porque funciona como el phantasma que "presenta" (entendido activamente como "hacer") un hecho o acontecimiento, y porque es un "representante" o "equivalente-de" [valant-pour] un hecho o acontecimiento. Esto es similar al campo de girasoles de van Gogh: éste no es una imagen de un campo real de girasoles; el cuadro de van Gogh "presenta" (en sentido activo) un objeto en tanto producción de un objeto ficticio y un contenido que "representa" un campo de girasoles parecido a los que se puede percibir en la realidad. En lugar de un esquema vertical, basado en una dicotomía entre lo real y su representación, en el cual se pasaría de un hecho o acontecimiento original a su representación narrativa, la "representancia" ofrece el esquema horizontal: tenemos en primer lugar una "mera presentación" como "aprehensión” [Auffassung] (Husserl), "interpretación” [Deutung] (Husserl) o "invención” (Ricoeur) que da lugar al phantasma del relato del hecho o acontecimiento, tal como fue establecido y testimoniado. En el segundo esquema, no se presupone 
una realidad plenamente articulada antes de nuestro contacto con el hecho o acontecimiento, excluyendo el hecho o acontecimiento real, sino que se establece un marco en el cual un "algo en general", que es "meramente presentado", se hace presente a la conciencia "como" tal hecho o tal acontecimiento.

Efectivamente, cuando se dice que la narración "no es" el acontecimiento — sería el género de lo Otro-, se trata de una negación duplicada, puesto que el acontecimiento en sí solo es en todo caso a través de una percepción, que ya implica un momento imaginativo. Por lo tanto, es posible afirmar que el acontecimiento en sí no es simplemente acontecimiento. He aquí como el género de lo Análogo permite pensar la diferencia en doble negación. Decir que la narración no es el acontecimiento (como primera negación) es duplicar otra negación; el acontecimiento en sí mismo no es un simple acontecimiento en tanto que conjunto de movimientos físicos: el acontecimiento "es" para la conciencia en la que se da. Dado que el evento no es idéntico a sí mismo en tanto que conjunto de movimientos físicos simples, sino por una conciencia en una percepción que ya es en sí misma imaginante [imaginante]; y dado que, a su vez, la narración implica un momento imaginante (el phantasma), es posible decir sin caer en una paradoja que el acontecimiento y la narración comparten este momento de mera presentación bajo la forma de una "aprehensión de sensaciones" (para la percepción) y de phantasmas (en la narración); y que el acontecimiento y la narración, en este sentido, son lo mismo. Pero esta "mismidad" es el resultado de un trabajo narrativo y no de un punto de partida (se relata algo solo después de lo que sucedió primero) ni de un postulado (los historiadores nos dicen qué se hizo en el pasado). Es a través del trabajo de lo Análogo que la narración es a la vez "un otro" que el acontecimiento y, no obstante, "lo mismo" que el acontecimiento.

Mediante la conciliación de la percepción con la imaginación, y dada su mediación común (sensaciones y phantasmas), se ha mostrado, en primer lugar, que la percepción se encuentra más articulada que una simple serie de sensaciones; en segundo lugar, que la imaginación ya se encuentra operando en la percepción (a fin de ir más allá de las simples sensaciones); y, por último, que el relato de 
un hecho o acontecimiento, por el soporte sintáctico, semántico y simbólico que les confiere, aporta un phantasma análogo a las sensaciones en la base de la percepción de ese hecho o acontecimiento. Esto complejiza así la analogía. La analogía ya no será entre la estructura del relato y la estructura del hecho o acontecimiento, sino entre la "aprehensión" de sensaciones y el phantasma de la narración. $\mathrm{Al}$ igual que el actor en escena desaparece como persona y crea, mediante su actuación, un phantasma que hace aparecer a Fausto o Lincoln, la narración es un trabajo que se borra como tal a fin de producir el artificio de un phantasma que hace aparecer "la verdad esencial" que Semprún y los otros sobrevivientes del campo de concentración quisieron preservar. Es solamente este phantasma el que podrá cautivar la imaginación de los lectores y así "mostrarles" lo que "verdaderamente pasó" en los campos ${ }^{56}$.

56. Traducido del francés por Ricardo Mendoza-Canales. En los casos en que no existe traducción castellana de las obras citadas, el traductor ha añadido a pie de página las citas originales en inglés y alemán. 

BIBLIOGRAFÍA 
